\title{
Preliminary Design Methodology for Hypersonic Engine Flowpaths
}

\author{
Sean M. Torrez*, James F. Driscoll ${ }^{\dagger}$, Derek J. Dalle*, Matthew L. Fotia* \\ Dept. of Aerospace Engineering, University of Michigan, Ann Arbor MI 48109 \\ Presented at the 16th AIAA/DLR/DGLR International Space Planes \\ and Hypersonic Systems and Technologies Conference, Bremen, Germany, October 2009
}

\begin{abstract}
A new scramjet engine model, called MASIV, has been developed for control-oriented applications. To reduce computational time, each component models the pertinent physical mechanisms while reducing the spatial dimensionality of the problem. New aspects of MASIV include real-gas dissociation, finite-rate chemistry, a new fuel-air mixing model, an assumed-PDF turbulent combustion model, and interactions of shocks and expansion waves.

Strategies for designing 2D scramjet inlets are discussed. One approach is optimize an inlet for a single flight condition. When an inlet designed in this way is at the design condition, all shocks intersect at the cowl leading edge. This optimizes performance at the design condition, but for off-design operation losses are highly sensitive to changes in Mach number and angle of attack. An improved inlet design is described that operates efficiently over a range of conditions. In addition, the scramjet combustor also is analyzed to show the effect of pressure distribution on thrust performance for five fuel injection locations. Results suggest general design guidelines, one of which is that injectors should be placed as far upstream as is practical, so that most of the combustion is completed upstream of the nozzle.
\end{abstract}

\section{Nomenclature}

$$
\begin{aligned}
A & =\text { cross-sectional area } \\
C & =\text { coefficient } \\
F & =\text { thrust } \\
H & =\text { vertical length scale } \\
L & =\text { horizontal length scale } \\
R & =\text { gas constant } \\
S & =\text { wall area } \\
T & =\text { temperature } \\
W & =\text { molecular weight } \\
Y & =\text { mass fraction } \\
\operatorname{Pr} & =\text { Prandtl number } \\
n & =\text { number of items of given type } \\
p & =\text { pressure }\left[\mathrm{N} / \mathrm{m}^{2}\right] \\
r & =\text { ratio } \\
x & =\text { horizontal spatial coordinate } \\
z & =\text { vertical spatial coordinate }
\end{aligned}
$$

\footnotetext{
${ }^{*}$ Graduate Student Research Assistant, AIAA Student Member

${ }^{\dagger}$ Professor of Aerospace Engineering, AIAA Fellow
} 


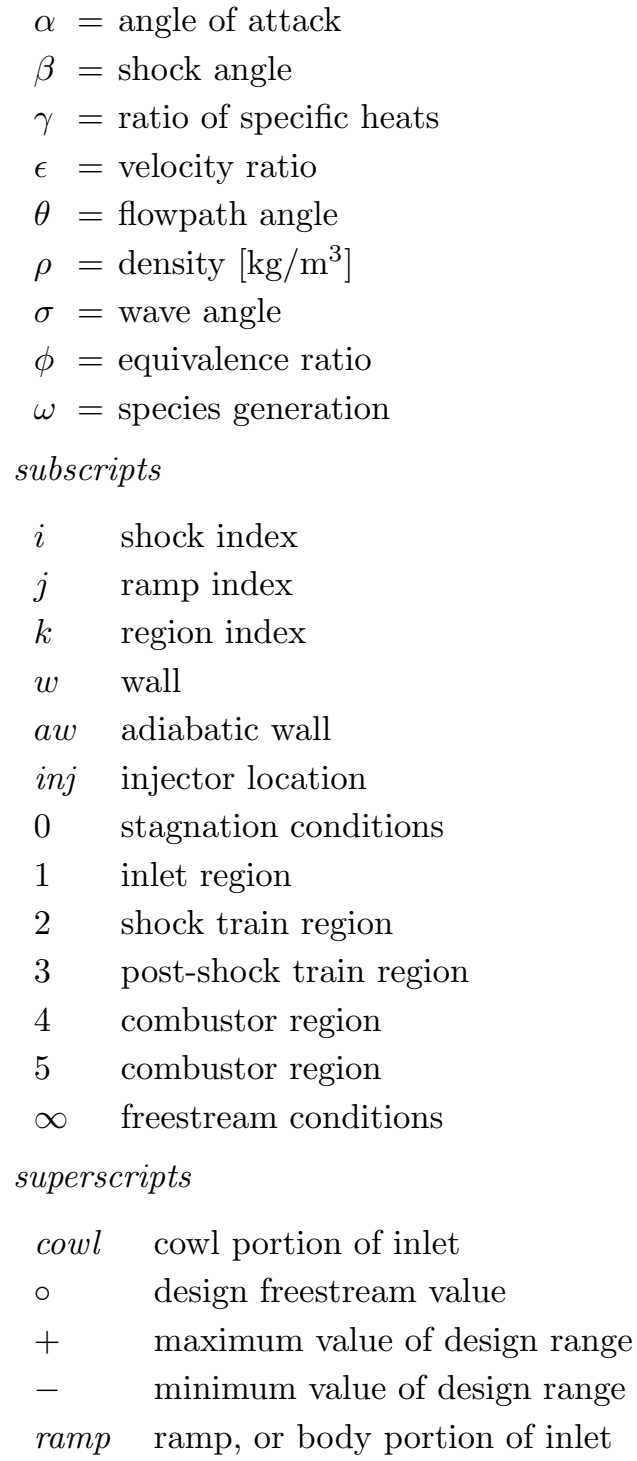

\section{Introduction}

In recent years there have been several efforts to simulate air-breathing hypersonic vehicles or their components. In particular, the model of Doman and Bolender ${ }^{1}$ is control-oriented, contains a number of control parameters, and includes reduced-order models in order to calculate all forces. It computes engine thrust, moments, and the trim conditions in a few seconds. The current effort provides a modeling framework with the potential to include actuators of interest, such as inlet cowl flap angle, inlet boundary layer bleed, inlet contraction ratio, fuel injector location, nozzle fuel injection, and plasma control of the flame stabilization location

An important component of the effort is the development of a submodel of the propulsion system that is both accurate and control-oriented. The result is the propulsion model called Michigan-AFRL Scramjetin-Vehicle (MASIV). The flow path must be specified a priori. The inlet is represented by a $2 \mathrm{D}$ geometry with multiple shock wave interactions, and the engine is represented by a 1D duct with area change, heat addition and friction. Fuel must be added from ports on the wall, but the user can select any number of ports, their locations, and the amount of fuel injected through each port.

To reduce the order of the turbulent combustion problem in the duct, it was important to leave only ordinary differential equations (ODEs) for the solution variables. This means that, for example, diffusion problems cannot be solved directly and their differential relationships must be replaced with algebraic laws. For a fuel jet burning in a cross flow, MASIV uses well-established scaling relations for turbulent mixing. 
These relations allow complex 3D combustion to be computed rapidly using a flamelet lookup table. Statistics of turbulence-chemistry interactions are included by employing probability distribution functions for each variable that has an effect on the rate of reaction. A ROM of the exhaust nozzle is included; it assumes that the nozzle flow is well-mixed so the $1 \mathrm{D}$ equations are combined with a finite-rate Arrhenius chemistry to compute the degree of gas recombination.

The reduced-order inlet model gives approximate solutions to the inviscid supersonic flow around 2D inlets. To do this, the code tracks shocks and expansion waves as they interact with each other and the inlet surface. Each wave is considered a boundary between two distinct but uniform regions. The result of this computation is a set of polygons in which the gas properties are uniform. This solution is not unlike the output of a finite-volume code, although the grid is generated automatically as the flow is solved. For a typical off-design condition, there are several hundred wave interactions within the inlet. Each of these interactions causes a loss in stagnation pressure, so the overall pressure recovery factor is significantly different than what was predicted previously using simple models that ignored wave interactions. MASIV simulates the wave interactions assuming a calorically imperfect gas (varying $c_{p}$ ) and constant chemical composition. Each expansion fan is discretized; an infinite number of weak waves is replaced by a number of finite-strength waves. The validity of each control-oriented component of the inlet, isolator and the combustor was assessed. ${ }^{2,3}$ MASIV results were compared to those computed with a high-fidelity 3D CFD code. $^{2}$

\section{Inlet design}

We propose a new method for inlet design that has several advantages for control-oriented applications. Several, for example Bogar et al. ${ }^{4}$ and Smart, ${ }^{5}$ discuss methods to design 2D scramjet inlets for a single flight condition (combination of $M_{\infty}$ and $\alpha$ ). However, inlets designed using this approach often show significantly decreased performance for even slight changes in flight conditions. ${ }^{3,6}$ On the other hand, integrated control simulations of hypersonic vehicles, by Bolendar and Doman, ${ }^{1}$ Chavez and Schmidt, ${ }^{7}$ and Frendreis et al. ${ }^{8}$ for example, have typically assumed an inlet with continuous sensitivity to flight conditions without discussing a realistic way to attain this performance.

To reconcile this difference, we propose a design method that produces inlets that guarantee smooth performance over a specified range of flight conditions. Instead of starting with only a single set of flight conditions, this method begins with two sets of flight conditions. The first set of conditions, the "+" conditions, consists of the maximum flight Mach number, $M_{\infty}^{+}$, and the minimum angle of attack, $\alpha^{+}$, while the "-" conditions consists of the minimum flight Mach number, $M_{\infty}^{-}$, and maximum angle of attack, $\alpha^{-}$. We call this range of flight conditions the design flight envelope.

The objective is to design a scramjet inlet with an overall compression ratio of $r_{p}=p_{2} / p_{\infty}$ using $n^{\text {ramp }}$ external ramps and $n^{\text {cowl }}$ internal turns. Examples of two such inlets are shown in Fig. 1. A well-designed inlet should have not only a high pressure recovery factor $\left(p_{0,2} / p_{0, \infty}\right)$ but also a continuous performance over a range of flight Mach numbers $\left(M_{\infty}\right)$ and angles of attack $(\alpha)$.

In previous analysis, ${ }^{3}$ we attempted to determine the physical phenomena that were causing the performance jumps of the previous inlet designs. To attain smooth performance, we apply a new set of constraints to the inlet design that prevents the jump-causing shock patterns from occurring. The improved design methodology is not an optimization but merely a method to determine an inlet that meets all of the constraints.

\section{A. Design methodology}

Although the primary goal of a scramjet inlet is to provide adequate compression to the combustor while minimizing the loss in stagnation pressure, there are several conditions that should be avoided. To demonstrate these unfavorable conditions, Fig. 2 the internal portion of the flow for the inlets in Fig. 1 at several flight conditions.

The first shock wave, which begins at the leading edge of the inlet, is called the bow shock. The leading edge of the cowl can also be called the lip. For a realistic inlet design, the bow shock should remain upstream of the lip, as in Figs. 2.a, 2.b, 2.e, and 2.f. If this is not the case, and the bow shock goes into the internal portion of the flow as in Figs. 2.c, 2.d, 2.g, and 2.h, the boundary layer is likely to separate. In addition, if the shock is incident on the cowl leading edge, there is a type IV shock interaction. ${ }^{9}$ Therefore our improved 


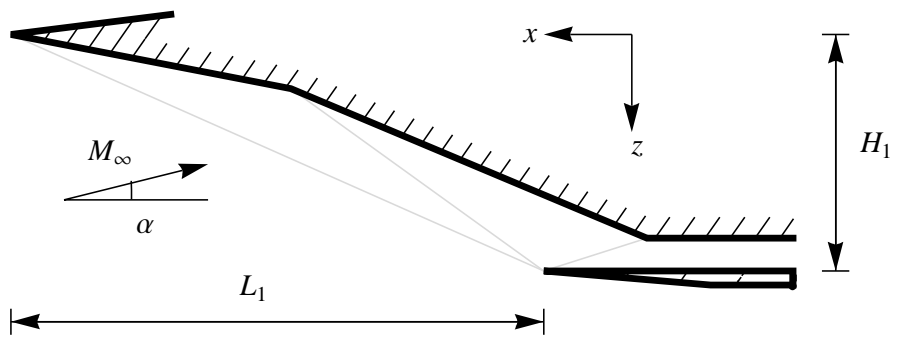

a) $n^{\text {ramp }}=2, n^{\text {cowl }}=1$

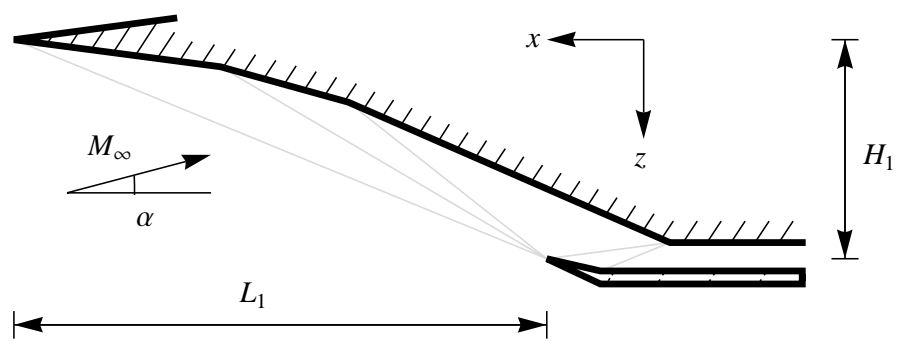

b) $n^{\text {ramp }}=3, n^{\text {cowl }}=2$

Figure 1. Examples of two scramjet inlets.

design method ensures that the bow shock remains upstream of the lip for the entire envelope of flight conditions.

We use the term cowl shock for the first internal shock, which begins at the lip or leading edge of the cowling. The cowl shock impinges on the main inlet body near another vertex, which we call the shoulder of the inlet. At this point the flow is turned to align more closely with the $x$-axis of the vehicle. Our design methodology requires that the cowl shock passes downstream of the shoulder before reflecting off of the main inlet body. In Figs. 2.a, 2.b, 2.e, and 2.f, the cowl shock reflects off of the main inlet body at a point upstream of the shoulder. This reflected shock is highly unfavorable because the flow entering the inlet passes through two shocks (the cowl shock and its reflection) before passing through the expansion at the shoulder. In Figs. 2.c, 2.d, 2.g, and 2.h, on the other hand, the flow passes through the cowl shock, then the expansion, and then the reflected shock. Because of the nonlinear nature of hypersonic shock waves, this difference in order has a noticeable effect on the inlet performance.

\section{B. Shock wave strengths}

Suppose that a pressure ratio, $r_{p}=p_{2} / p_{\infty}$, and two flight conditions, $M_{\infty}^{+}, \alpha^{+}$and $M_{\infty}^{-}, \alpha^{-}$, are given. We design an inlet for which the compression ratio is at least $r_{p}$ over the entire design range. We introduce a notation that $M_{1, i}^{+}$is the Mach number before the $i$ th shock assuming the "+" flight conditions.

Set $M_{1,1}^{+}=M_{\infty}^{+}$and select a value of $\beta_{1}^{+}$. Because the pressure losses tend to be higher at larger Mach numbers, we constrain the external shocks to have equal normal Mach numbers for the "+" conditions, so

$$
M_{1, i+1}^{+} \sin \beta_{i+1}^{+}=M_{1, i}^{+} \sin \beta_{i}^{+}
$$

for $i=1, \ldots, n^{\text {ramp }}-1$. The deflection angle for each external shock is determined using

$$
\tan \delta_{i}=2 \cot \beta_{i}^{+} \frac{\left(M_{1, i}^{+}\right)^{2} \sin ^{2} \beta_{i}^{+}-1}{\left(M_{1,1}^{+}\right)^{2}\left(\gamma+\cos 2 \beta_{i}^{+}\right)+2}
$$

for $i=1, \ldots, n^{r a m p}$, and the post-shock Mach number is

$$
M_{1, i+1}^{+}=\frac{1}{\sin \left(\beta_{i}^{+}-\delta_{i}\right)} \sqrt{\frac{1+\frac{\gamma-1}{2}\left(M_{1, i}^{+}\right)^{2} \sin ^{2} \beta_{i}^{+}}{\gamma\left(M_{1, i}^{+}\right)^{2} \sin ^{2} \beta_{i}^{+}-\frac{\gamma-1}{2}}}
$$




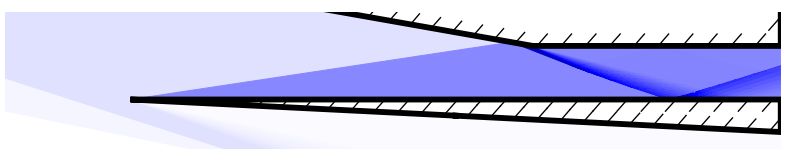

a) $M_{\infty}=7.0, \alpha=0^{\circ}$

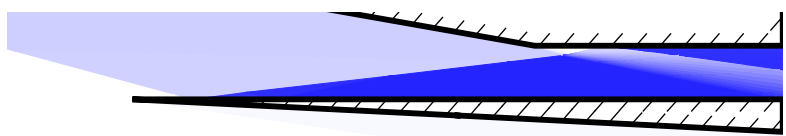

c) $M_{\infty}=9.0, \alpha=0^{\circ}$

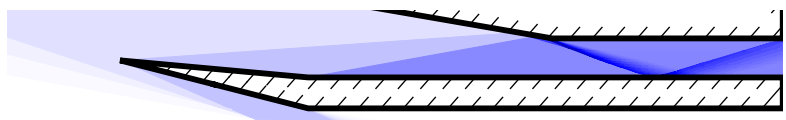

e) $M_{\infty}=7.0, \alpha=0^{\circ}$

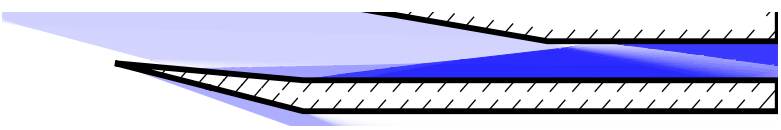

g) $M_{\infty}=9.0, \alpha=0^{\circ}$

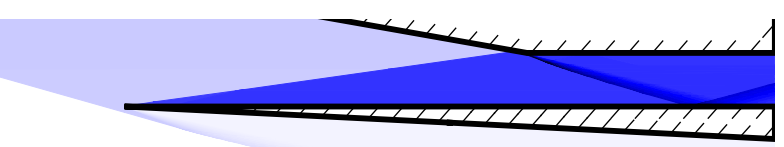

b) $M_{\infty}=8.0, \alpha=+2^{\circ}$

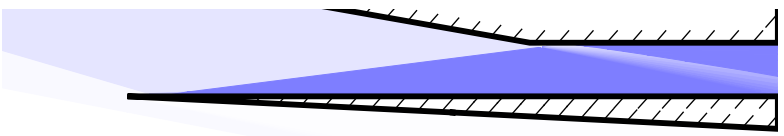

d) $M_{\infty}=8.0, \alpha=-2^{\circ}$

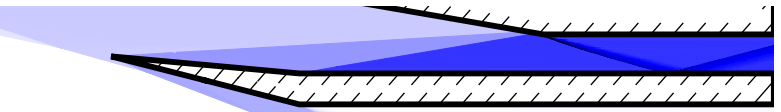

f) $M_{\infty}=8.0, \alpha=+2^{\circ}$

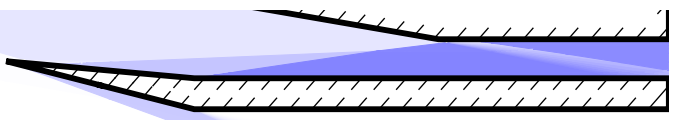

h) $M_{\infty}=8.0, \alpha=-2^{\circ}$

Figure 2. Examples of unfavorable inlet performance. Darker shades of blue represent higher non-dimensional pressures; black represents $p / p_{\infty}=60$. Notice the similar effect on the shock pattern from increasing $M_{\infty}$ and decreasing $\alpha$.

for $i=1, \ldots, n^{r a m p}$. The deflection angles, $\delta_{i}=\theta_{1, i+1}-\theta_{1, i}$ where $\theta_{1, i}$ is the angle between the flow and the $x$-axis before the $i$ th shock, do not have a superscript because they are part of the geometry and do not depend on the flight conditions. Thus the shock angles for the "-" conditions can be determined using Eq. 2 only replacing the "+" superscripts with "-" superscripts. Then the compression ratio across each shock is

$$
r_{i}=\frac{2 \gamma}{\gamma+1}\left(M_{1, i}^{-}\right)^{2} \sin ^{2} \beta_{i}^{-}-\frac{\gamma-1}{\gamma+1}
$$

for $i=1, \ldots, n^{r a m p}$. We apply the compression constraint as

$$
r_{p}=\prod_{i=1}^{n} r_{i}=r_{n}^{n^{\text {cowl }}} \prod_{i=1}^{n^{\text {ramp }}} r_{i}
$$

where $r_{i}$ has the same value when $i=n^{\text {ramp }}+1, \ldots, n$. For the $n^{\text {cowl }}$ internal shocks, we determine the shock angles using

$$
\sin \beta_{i}^{-}=\frac{1}{M_{i}^{-}} \sqrt{\frac{\gamma+1}{2 \gamma} r_{n}-\frac{\gamma-1}{2 \gamma}}
$$

and the internal deflection angles are found using Eq. 2. Then we iteratively determine the value of $\beta_{1}^{+}$that leads to a sequence of deflection angles that satisfy the constraint

$$
\alpha^{+}+\sum_{i=1}^{n^{\text {ramp }}} \delta_{i}=\sum_{i=n^{\text {ramp }}+1}^{n} \delta_{i}
$$

\section{Geometric solution}

Once we have determined the shock angles and deflection angles, we can determine the geometry of the inlet. In order to increase the mass flow to the combustor, we position the cowl so that the bow shock exactly on the lip for the "+" conditions. To avoid shock interactions, we also constrain that the remaining external shocks must intersect the leading edge of the cowl, and thus the geometry is uniquely determined. 
To satisfy the zero-spillage constraint, the position of the cowl leading edge must satisfy

$$
\tan \left(\beta_{1}^{+}-\alpha^{+}\right)=\frac{H_{1}}{L_{1}}=\frac{z_{1,1}^{\text {ramp }}-z_{1,1}^{\text {cowl }}}{x_{1,1}^{\text {ramp }}-x_{1,1}^{\text {cowl }}},
$$

and the remaining points on the external portion of the inlet must satisfy

$$
\begin{aligned}
& x_{1, i}^{\text {ramp }}=\frac{z_{1,1}^{\text {cowl }}+x_{1,1}^{\text {cowl }} \tan \sigma_{i}^{+}-z_{1, i-1}^{\text {ramp }}-x_{1, i-1}^{\text {ramp }} \tan \theta_{1, i}}{\tan \sigma_{i}^{+}-\tan \theta_{1, i}} \\
& z_{1, i}^{\text {ramp }}=z_{1, i}^{\text {ramp }}+\left(x_{1, i-1}^{\text {ramp }}-x_{1, i}^{\text {ramp }}\right) \tan \theta_{1, i}
\end{aligned}
$$

where $\sigma_{i}^{+}=\theta_{1, i}+\beta_{i}^{+}$for $i=1, \ldots, n^{\text {ramp }}-1$. The scale of the inlet does not affect the design algorithm, so we set $L_{1}=1$. Also, we put the leading edge at the point $x_{1,1}^{r a m p}=z_{1,1}^{\text {ramp }}=0$.

The first cowl shock must not go upstream of the shoulder, so the coordinates of the first shoulder are

$$
\begin{aligned}
x_{1, i}^{\text {ramp }} & =\frac{z_{1, i-1}^{\text {ramp }}-x_{1, i-1}^{\text {ramp }} \tan \sigma_{i}^{-}-z_{1, j}^{\text {cowl }}-x_{1, j}^{\text {cowl }} \tan \theta_{1, i}}{\tan \sigma_{i}^{-}+\tan \theta_{1, i}} \\
z_{1, i}^{\text {ramp }} & =z_{1, i-1}^{\text {ramp }}+\left(x_{1, i-1}^{\text {ramp }}-x_{1, i}^{\text {ramp }}\right) \tan \theta_{1, i}
\end{aligned}
$$

where $i=n^{\text {ramp }}+1$. For the remaining internal shocks, if $n^{\text {cowl }}>1$, there are two constraints. In Figs. $2 \mathrm{~g}$ and $2 \mathrm{~h}$, the two internal shocks intersect each other. When this happens, the two shocks combine, and the benefit of having multiple internal shocks is eliminated. Therefore we determine the locations of the shoulders so that the $i$ th shock never goes downstream of the vertex $\left(x_{1, i+1}^{\operatorname{ramp}}, y_{1, i+1}^{\mathrm{ramp}}\right)$. The second constraint is that none of the internal shocks reflect upstream of the shoulders. The coordinates of the remaining vertices are given by

$$
\begin{aligned}
x_{1, i}^{\text {ramp }} & =\frac{z_{1, i-1}^{\text {ramp }}+x_{1, i-1}^{\text {ramp }} \tan \theta_{1, i}-z_{1, j-1}^{\text {cowl }}-x_{1, j-1}^{\text {cowl }} \tan \sigma_{i-1}^{+}}{\tan \sigma_{i-1}^{+}+\tan \theta_{1, i}} \\
z_{1, i}^{\text {ramp }} & =z_{1, i-1}^{\text {ramp }}+\left(x_{1, i-1}^{\text {ramp }}-x_{1, i}^{\text {ramp }}\right) \tan \theta_{1, i} \\
x_{1, j}^{\text {cowl }} & =\frac{z_{1, j-1}^{\text {cowl }}-x_{1, j-1}^{\text {ramp }} \tan \theta_{1, i}-z_{1, i}^{\text {ramp }}+x_{1, i}^{\text {ramp }} \tan \sigma_{i}^{-}}{\tan \sigma_{i}^{-}-\tan \theta_{1, i}} \\
z_{1, j}^{\text {cowl }} & =z_{1, j-1}^{\text {cowl }}+\left(x_{1, j}^{\text {cowl }}-x_{1, j-1}^{\text {cowl }}\right) \tan \theta_{1, i}
\end{aligned}
$$

for $i=n^{\text {ramp }}+2, \ldots, n$ where $j=i-n^{\text {ramp }}$ and $\sigma_{i}^{-}=\theta_{1, i}-\beta_{i}^{-}$.

Unlike the single-condition design of Smart, ${ }^{5}$ this robust design does not satisfy any optimality conditions. However, our inlets do have realistic properties that we believe are important for a scramjet inlet. As an interesting note, our design methodology will produce the traditional single-condition design if $M_{\infty}^{+}=M_{\infty}^{-}$ and $\alpha^{+}=\alpha^{-}$.

\section{Results}

We compare the performance of two inlets using the design methodology described in the previous subsections. The first inlet is a single-condition design, and the second inlet design is intended to work over a range of flight Mach numbers. The design conditions are shown in Table 1. Both inlets have a compression ratio of $p_{2} / p_{\infty}=52$ when $M_{\infty}=8.0$ and $\alpha=0$. The coordinates of the vertices of the improved inlet are given in Table 2 .

Table 1. Design flight conditions for two inlets

\begin{tabular}{lcccc}
\hline \hline & $M_{\infty}^{-}$ & $\alpha^{-}$ & $M_{\infty}^{+}$ & $\alpha^{+}$ \\
\hline single-condition design & 8.0 & 0.0 & 8.0 & 0.0 \\
improved design & 7.0 & 0.0 & 9.0 & 0.0 \\
\hline \hline
\end{tabular}

We use a supersonic wave-modeling program created by the authors ${ }^{2,3}$ to analyze the performance of the inlets. 
Table 2. Scaled coordinates for improved inlet design

\begin{tabular}{cccc}
\hline \hline & $k$ & $\left(x_{1,1}^{\text {ramp }}-x_{1, k}\right) / H_{1}$ & $\left(z_{1, k}-z_{1,1}^{\text {ramp }}\right) / H_{1}$ \\
\hline ramp & 1 & 0 & 0 \\
& 2 & 3.1921 & 0.2382 \\
& 3 & 4.7058 & 0.4830 \\
& 4 & 6.5702 & 0.9784 \\
& 5 & 6.7037 & 0.9971 \\
cowl & 1 & 5.9910 & 1 \\
& 2 & 6.4102 & 1.05866 \\
\hline \hline
\end{tabular}

Fig. 3 shows the flow through the improved inlet for the same conditions as in Fig. 2. Compared to Fig. 2e-f, the internal shocks in Fig. 3 do not intersect each other, and they do not move upstream of the appropriate shoulders.

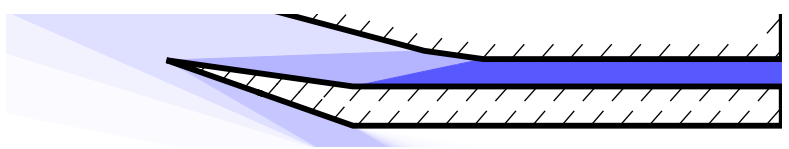

a) $M_{\infty}=7.0, \alpha=0^{\circ}$

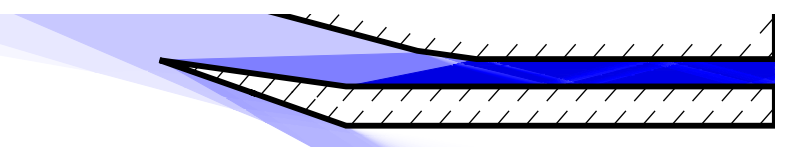

b) $M_{\infty}=8.0, \alpha=+2^{\circ}$

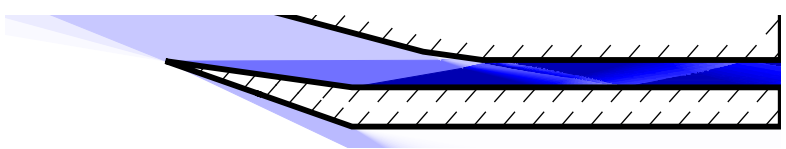

c) $M_{\infty}=9.0, \alpha=0^{\circ}$

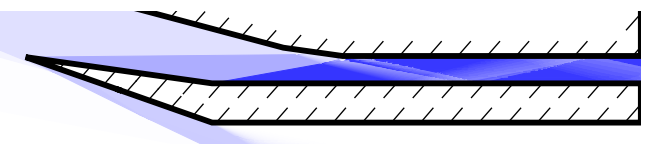

d) $M_{\infty}=8.0, \alpha=-2^{\circ}$

Figure 3. Flow pattern for improved inlet design with $n^{\text {ramp }}=3$ and $n^{\text {cowl }}=2$. Darker shades of blue represent higher non-dimensional pressures; black represents $p / p_{\infty}=120$.

Fig. 4 shows the performance of the two inlets for a range of flight Mach numbers and angles of attack. For flight Mach numbers that are not in the immediate vicinity of 8.0, the improved design has a much higher pressure recovery factor. More importantly, the sensitivity to small changes in flight condition is greatly decreased for the improved inlet. In particular, the single-condition design has a very good pressure recovery factor at $M_{\infty}=8.0$ and $\alpha=0$, but the pressure recovery factor drops by more than $10 \%$ for small changes in flight Mach number. The improved inlet, on the other hand, has a lower pressure recovery factor at that particular flight condition, but the performance is not subject to sharp decreases.

The smooth performance curves of the improved inlet have two major applications. First, vehicles with lower sensitivities are much easier to control. Second, the inlet can be used over a range of flight conditions. This inlet could be used, for example, to accelerate from $M_{\infty}=7.0$ to $M_{\infty}=9.0$. In general, the improved design methodology leads to inlets that can perform a much wider range of missions.

\section{Combustor Design}

A good combustor design is subject to different constraints depending on its operating point for this type of vehicle. Experimental evidence ${ }^{10,11}$ suggests that combustion properties, such as total heat release and heat release rate are highly dependent on upstream conditions in the ramjet case. The present study deals with the scramjet case, for which there is less experimental evidence available. Full-fidelity computer simulations (CFD) serve as the bridge between available experimental data and quantities that are difficult or impossible to measure in the lab but that are useful for development of reduced order models (ROMs).

\section{A. Design methodology}

In the combustor, the main goal is to combust the fuel in the way that produces the most thrust. However, just as in the inlet, there are some other concerns that affect the design choices. The location of fuel injection 


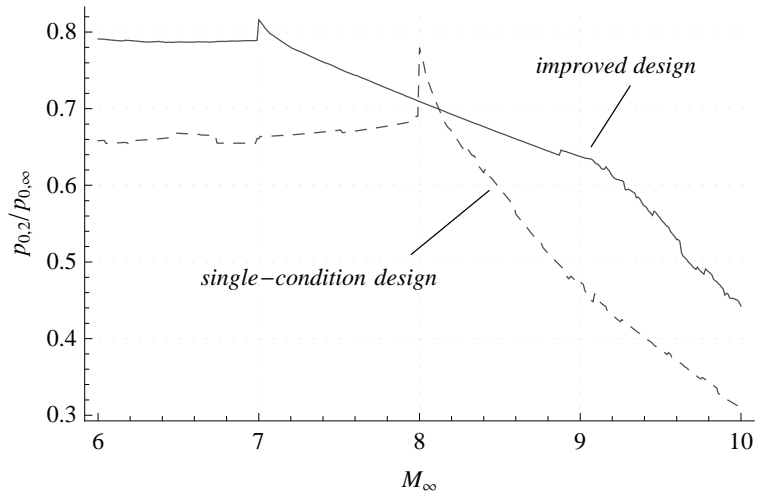

a) $\alpha=0$

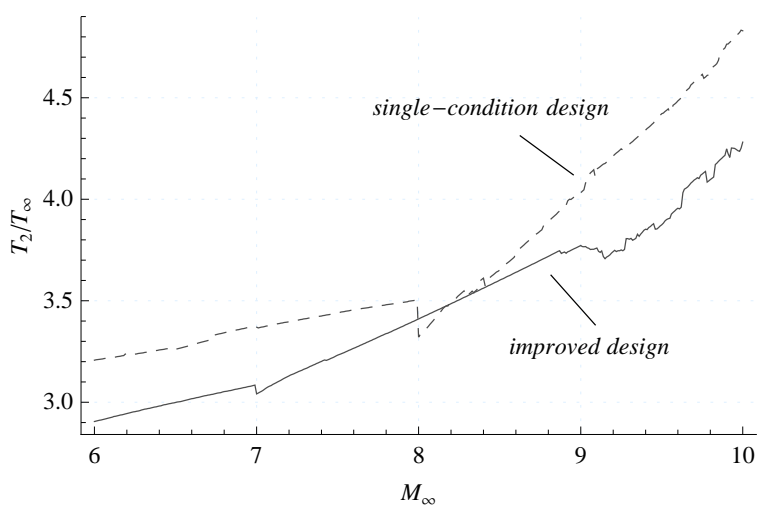

c) $\alpha=0$

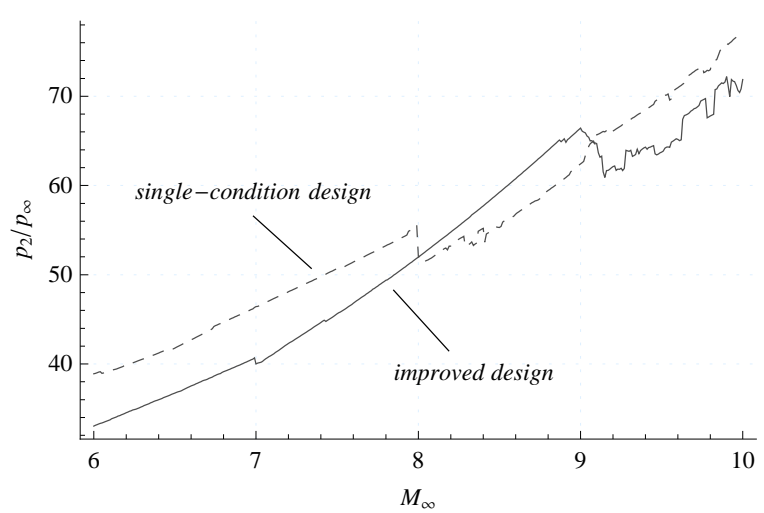

e) $\alpha=0$

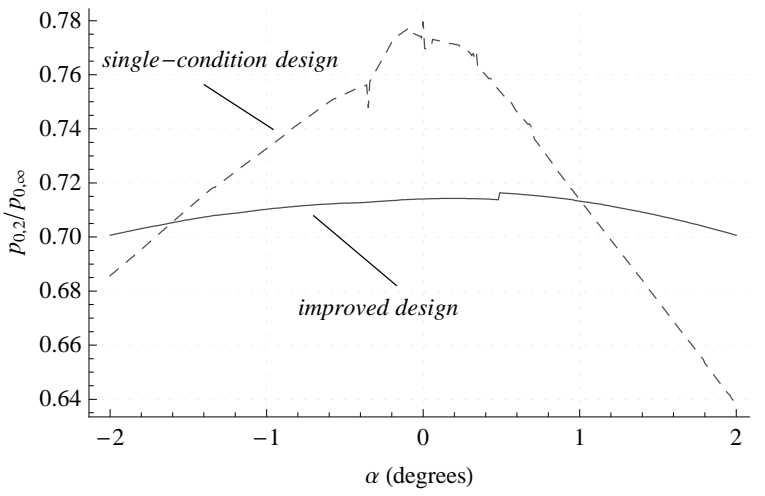

b) $M_{\infty}=8.0$

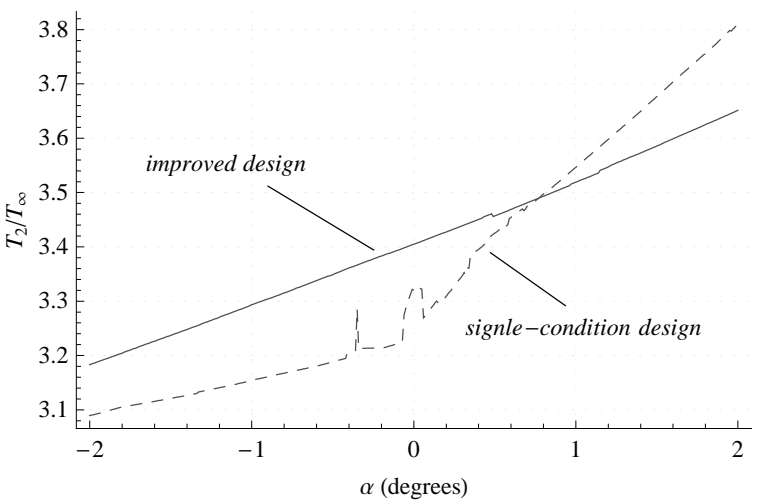

d) $M_{\infty}=8.0$

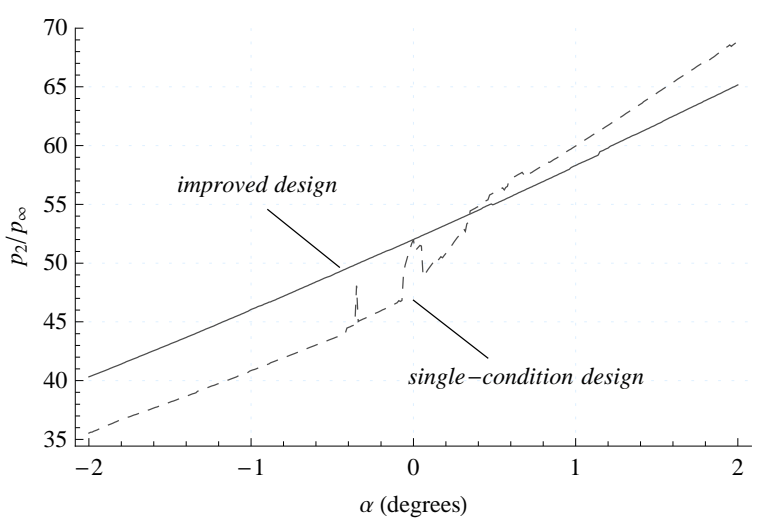

f) $M_{\infty}=8.0$

Figure 4. Performance of two scramjet inlet designs. The compression ratio is $p_{2} / p_{1}=52$ at $M_{\infty}=8.0$ and $\alpha=0$. 
is important because the duct geometry has a large effect on how fuel is released. In the ram mode, the location of heat release affects where and when the duct will choke, which affects the total heat release and its distribution, which in turn changes the thrust and the moment the engine causes. In the scram mode, the location of the heat addition cannot affect upstream conditions since the flow is supersonic everywhere, but it does still affect the thrust and moment caused by the engine. The ram mode will be discussed in future work.

It is best to burn the fuel at the highest available pressure, which means that the constant-area section of the duct is the best place to inject fuel. This can be seen clearly in Fig. 5. In Fig. 5, we see that as the injector moves further back, more of the combustion takes place in the expanding section of the duct and the pressure achieved is not as high as when the combustion occurs primarily in the constant-area section. Since combustion causes a pressure rise in a supersonic flow ${ }^{12}$ and the pressure ratio is determined by the amount of fuel injected, for a constant equivalence ratio, $\phi$, we expect to have the maximum pressure rise for a larger initial pressure. Thus, Fig. 7, which shows the effect of varying isolator exit pressure. Again, as the injector is moved further aft, the thrust decreases because less pressure is available to the nozzle section.

\section{B. Combustion modeling}

The MASIV model, developed at the University of Michigan, ${ }^{2,3,13,14}$ solves for the heat release distribution for both subsonic and supersonic flows, which is used to compute the thrust of hypersonic vehicle engines (typically ramjets or scramjets).

The combustor code solves a set of differential and algebraic equations in space, ${ }^{15}$ marching axially through the combustion duct. Since combustion in most engines is mixing-limited rather than reaction ratelimited, jet mixing must be computed. However, since differential equations are allowed only with respect to the axial distance coordinate, an algebraic jet spreading profile must be used; ${ }^{16}$ the solution is based on flow field similarity. The model considers finite-rate chemistry via the Stationary Laminar Flamelet Model (SLFM) ${ }^{17}$ which considers each point in the flame and maps it to the solution of a corresponding counterflow flamelet. Because this is a probability distribution function (PDF) approach, it includes the effects of different strain fields, species diffusion, and momentum diffusion, as well as turbulence, as the duct velocity and fuel jet velocity change.

The MASIV code marches the flow conservation equations downstream. All flow states are allowed to vary in the downstream axial direction, defined $-\hat{x}$. Derivatives are allowed with respect to the axial coordinate only. Some quantities, such as jet spreading and mixing, vary in the transverse directions, but they may only vary algebraically, such that their evolutions do not depend on the information propogating downstream. The equations solved in the code are presented in Tables 3 and 4 .

Table 3. Differential equations.

\begin{tabular}{|c|c|}
\hline ODE Name & Equation \\
\hline Equation of State & $\frac{1}{p} \frac{d p}{d x}=\frac{1}{T} \frac{d T}{d x}+\frac{1}{\rho} \frac{d \rho}{d x}-\frac{1}{W} \frac{d W}{d x}$ \\
\hline Cons. of Mass & $\frac{1}{\rho} \frac{d \rho}{d x}=\frac{1}{\dot{m}} \frac{d \dot{m}}{d x}-\frac{1}{u} \frac{d u}{d x}-\frac{1}{A} \frac{d A}{d x}$ \\
\hline Cons. of Species & $\frac{d Y_{i}}{d x}=\frac{\dot{\omega}}{\rho u}+\frac{1}{\dot{m}} \frac{d \dot{m}_{i}}{d x}-\frac{Y_{i}}{\dot{m}} \frac{d \dot{m}}{d x}$ \\
\hline Cons. of Momentum & $\frac{1}{u} \frac{d u}{d x}=-\frac{1}{\rho u^{2}} \frac{d p}{d x}-\frac{C_{f}}{2 A} \frac{d S_{w}}{d x}-\frac{(1-\varepsilon)}{\dot{m}} \frac{d \dot{m}}{d x}$ \\
\hline Cons. of Energy & $\begin{aligned} \frac{1}{h_{0}} \frac{d T}{d x} \sum_{i} c_{p, i} Y_{i}= & -\frac{u}{h_{0}} \frac{d u}{d x}-\frac{1}{\dot{m}} \frac{d \dot{m}}{d x}+\frac{1}{h_{0} \dot{m}} \frac{\rho u C_{f}\left(h_{a w}-h_{w}\right)}{2 P r^{2 / 3}} \frac{d S_{w}}{d x} \cdots \\
& +\frac{1}{h_{0} \dot{m}} \frac{\rho u^{3} C_{f}}{2} \frac{d S_{w}}{d x}-\frac{1}{h_{0}} \sum_{i} h_{i} \frac{d Y_{i}}{d x}+\frac{1}{h_{0} \dot{m}} \sum_{i} h_{i} \frac{d \dot{m}_{i}}{d x}\end{aligned}$ \\
\hline
\end{tabular}

A discussion of how the chemistry and the conservation equations are solved is presented in the cited works by Torrez et al. and will not be repeated here. Verification of the MASIV model is ongoing. We verify by comparing directly to experiments conducted at the University of Michigan and by comparing to full-fidelity CFD of the same experimental configuration. 


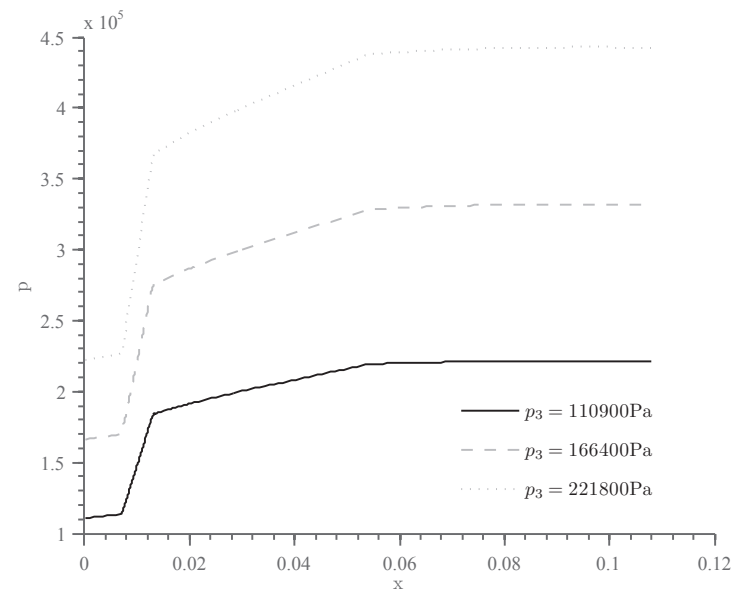

a) $p$ vs. $x, x_{i n j}=0.01 \mathrm{~m}$

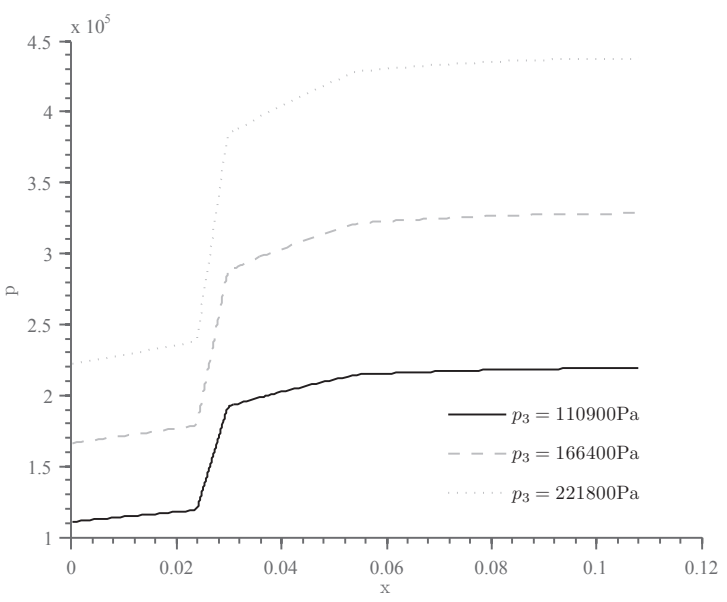

c) $p$ vs. $x, x_{i n j}=0.0268 m$

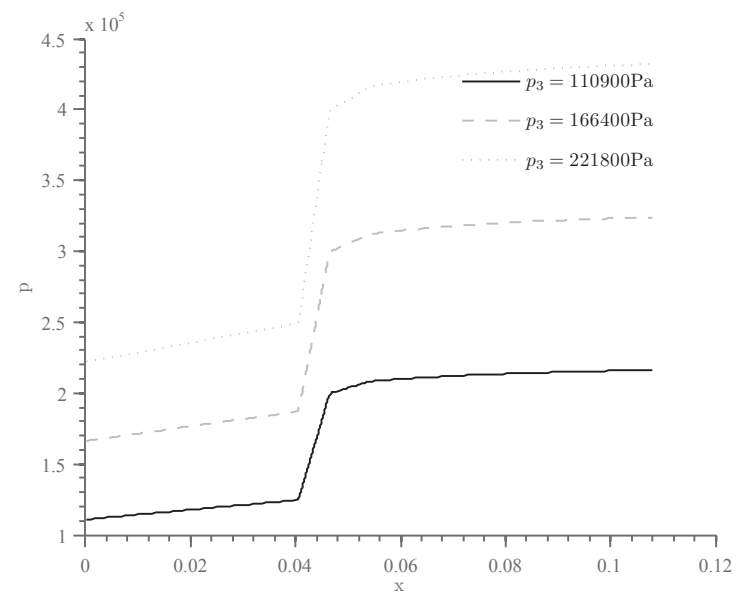

e) $p$ vs. $x, x_{i n j}=0.0435 \mathrm{~m}$

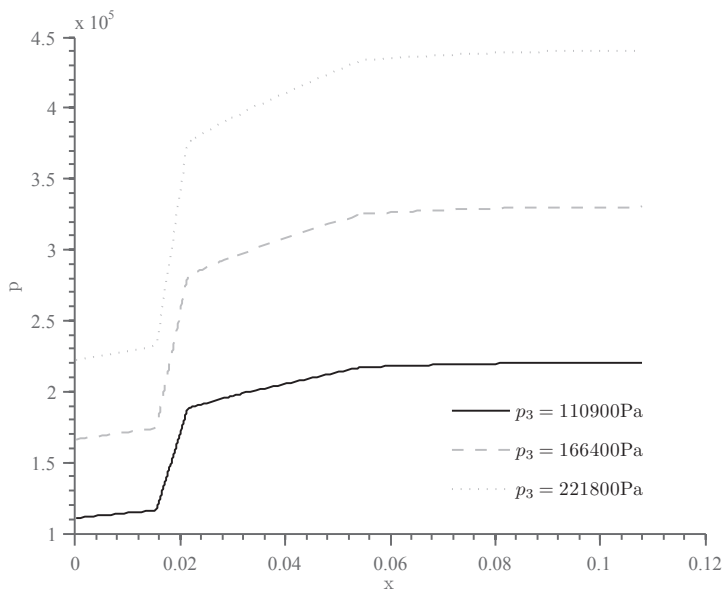

b) $p$ vs. $x, x_{i n j}=0.0184 \mathrm{~m}$

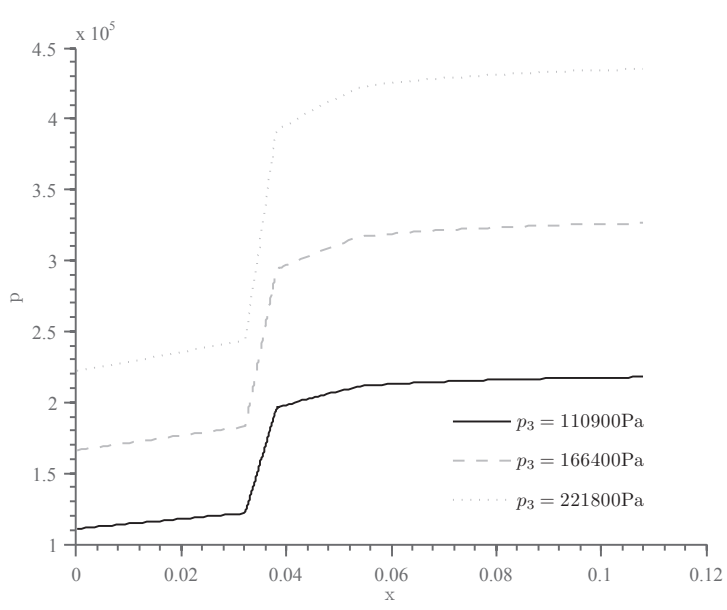

d) $p$ vs. $x, x_{i n j}=0.0352 m$

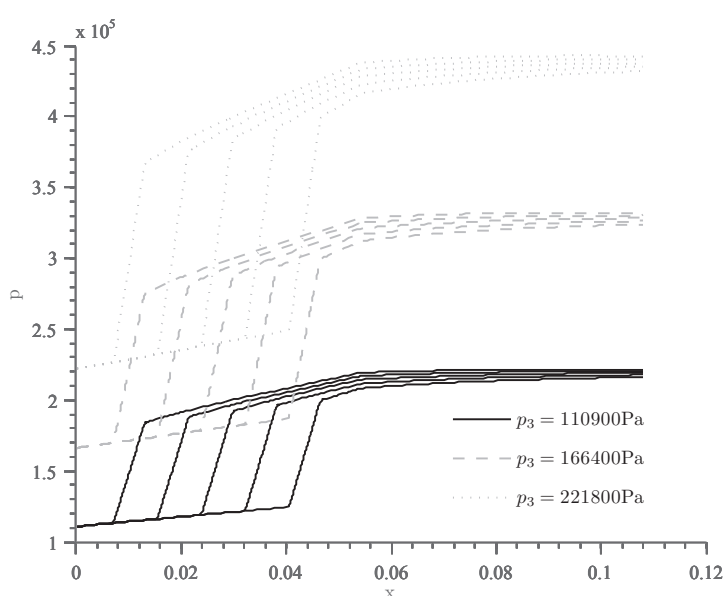

f) $p$ vs. $x$, all plots overlayed.

Figure 5. Pressure evolution as a function of axial distance, $x$ with varying isolator exit pressure, $p_{3}$. Each plot has a different fuel injection location, with downstream distance increasing in each successive plot. 
Table 4. Additional equations.

\begin{tabular}{|c|c|c|}
\hline Source & Variable & Equation \\
\hline \multirow{5}{*}{ From Solution } & $p$ & $=\int \frac{d p}{d x} d x$ \\
\hline & $\rho$ & $=\int \frac{d \rho}{d x} d x$ \\
\hline & $Y_{i}$ & $=\int \frac{d Y_{i}}{d x} d x$ \\
\hline & $u$ & $=\int \frac{d u}{d x} d x$ \\
\hline & $T$ & $=\int \frac{d T}{d x} d x$ \\
\hline \multirow{6}{*}{ Given } & $A$ & $=A(x)$ \\
\hline & $\frac{d A}{d x}$ & $=\frac{d A}{d x}(x)$ \\
\hline & $\begin{array}{l}d x \\
\frac{d \dot{m}_{i}}{d x}\end{array}$ & $=\frac{d \dot{m}_{i}}{d x}(x)$ \\
\hline & $\dot{\omega}$ & $=\dot{\omega}(x)$ \\
\hline & $W_{i}$ & $=$ const.$^{\dagger}$ \\
\hline & $C_{f}$ & $=$ const \\
\hline \multirow{10}{*}{ Computed } & $\dot{m}$ & $=\rho u A$ \\
\hline & $\frac{d W}{d x}$ & $=-W^{2} \sum_{i}\left(\frac{1}{W_{i}} \frac{d Y_{i}}{d x}\right)$ \\
\hline & $\frac{d S_{w}}{d x}$ & $=\sqrt{16 A+\left(\frac{d A}{d x}\right)^{2}}$ \\
\hline & $\frac{d \dot{m}}{d x}$ & $=\sum_{i} \frac{d \dot{m}_{i}}{d x}$ \\
\hline & $h_{i}$ & $=h_{i}(T)^{\dagger}$ \\
\hline & $h$ & $=\sum_{i} h_{i}$ \\
\hline & $h_{0}$ & $=h+\frac{u^{2}}{2}$ \\
\hline & $h_{w}$ & $=h\left(T_{w}\right)$ \\
\hline & $h_{a w}$ & $=h+\sqrt{\operatorname{Pr} \frac{u^{2}}{2}}$ \\
\hline & $a$ & $=\frac{\partial h}{\partial x} /\left(\frac{\partial h}{\partial x}-T \frac{\partial R}{\partial x}-R \frac{\partial T}{\partial x}\right)$ \\
\hline
\end{tabular}

${ }^{\dagger}$ from CHEMKIN

\section{Results}

For the scram case, it appears that positioning the injector as far from the nozzle as possible yields best results. This coincides with our intuition about combustors, because it gives a long residence time and as complete combustion as is possible before the fluid enters the nozzle. It is important to note that this conclusion is only valid for the high scram mode, since in this mode there is little possibility of thermally choking the flow by adding too much fuel. The ram case will be much more complicated in terms of design, and we believe that the ram case will require several injectors, possibly with one in the diverging part of the duct (the internal nozzle).

The duct used in this study is shown in Fig. 6. This duct is based on the combustor used in the University of Michigan Dual-Mode Combustor experiment discussed in [10], although it has some slightly different properties. The diverging section is considered to be an internal nozzle, and 5 different fueling locations are considered, as marked in the diagram. Note that the drawing is not to scale.

The results of the design study on pressure, $p_{3 a}$, and temperature, $T_{3 a}$, variations are shown in Fig. 7. Note that the thrust increases in general as the isolator exit pressure is increased (which is expected), but that when the injector is moved further back, the pressure rise occurs at a less and less favorable location, since more of the combustion happens in the nozzle.

Note that the scales of the pressure and temperature variation plots are far different. However, the curves for temperature fall very nearly on top of one another, suggesting that the effect of temperature is the same regardless of injector location. One reason for this is that since we normalize the thrust by the available capture momentum, the effect of decresing density, $\rho_{3 a}$, due to increasing temperature while 


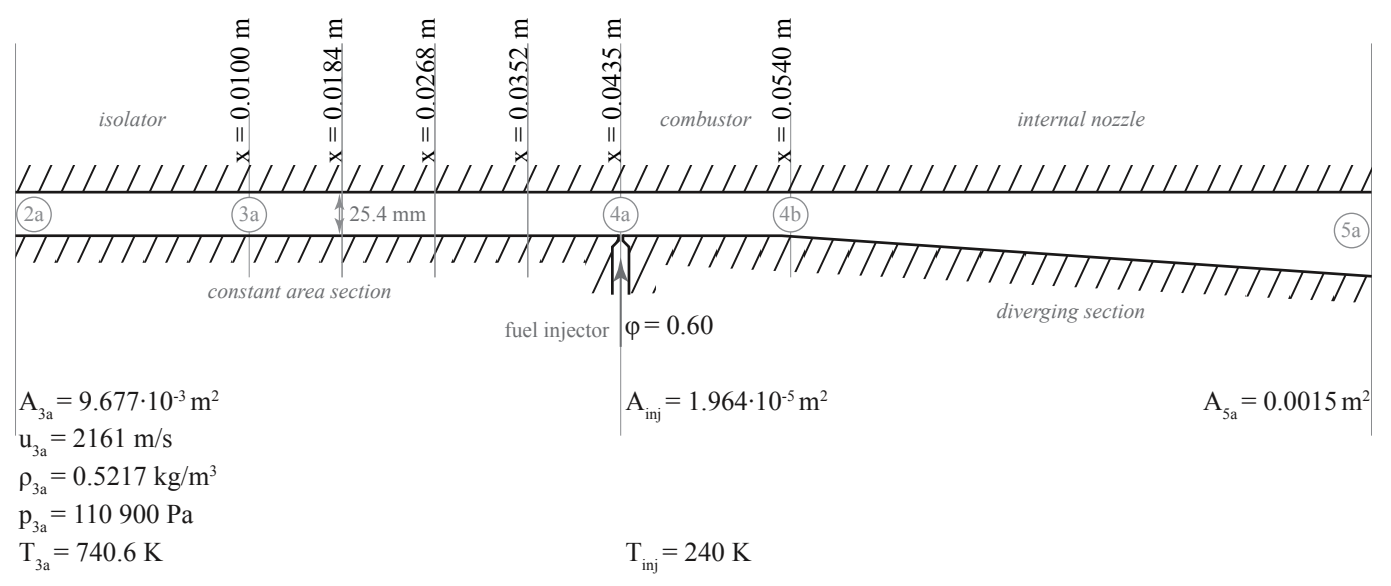

Figure 6. Simplified duct geometry for simulation.

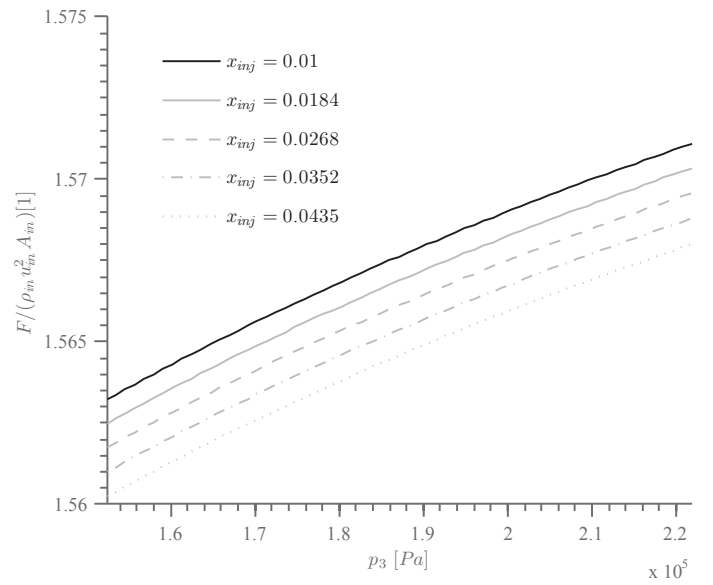

a) $F$ vs. $p_{3 a}$

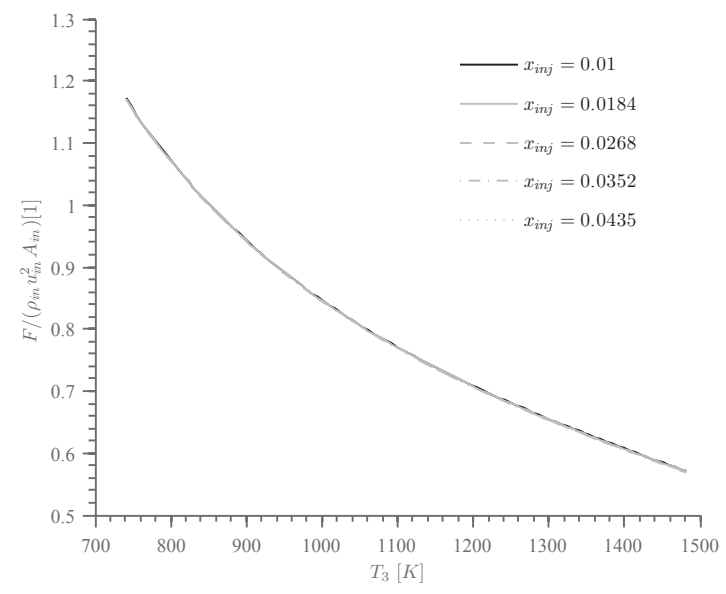

b) $F$ vs. $T_{3 \mathrm{a}}$

Figure 7. Thrust as a function of $p_{3 a}$ and $T_{3 a}$ for a variety of different fuel injector locations. 
holding pressure constant, is removed. The other reason is that since this computation has fixed equivalence ratio (even though the mass flow rate through the engine changes with the pressure or the temperature variations), the chemistry is only a function of the state of the air stream and the state of the fuel stream.

The flamelet algorithm requires that lookup tables be generated for all combustor inlet pressures and temperatures of interest. To demonstrate the feasibility of this approach, the lookup tables were generated for the exact chemistry, but for only a limited range of pressures and temperatures. Future approaches will include a large number of chemistry files to account for all these variations. A higher temperature or pressure upstream of fuel injection will encourage more complete combustion with a corresponding higher rate of heat addition and increased thrust.

\section{Conclusions}

Using a reduced-order model, we have demonstrated that if the propulsion system is designed to be optimized for a single operating condition, large losses will occur at slightly off-design conditions. A better approach is to design the inlet geometry, fuel injection locations, and other parameters to give optimum performance over a range of flight Mach numbers and angles of attack. Although operation over a wide envelope of flight conditions is challenging for any aerospace vehicle, this is particularly true for hypersonic vehicles. In hypersonic flight, strong shock waves are generated, and the performance can be very sensitive to the locations of these shock waves.

An inlet design is introduced that can operate over a range of flight conditions without showing sharp decreases in performance. To achieve this property, some performance is sacrificed at cruise conditions. However the cruise-condition performance is regained with a narrower design flight envelope. In other words, it is possible with this method to design an inlet that can operate over a wide range of conditions or an inlet that is very efficient at a single condition but does not have a large sensitivity to angle of attack.

In this discussion we considered only passive inlets, although the design methodology should be applicable to inlets that have geometry controlled in some way. In general we found that the key to continuous performance is preventing shocks from interacting with each other or crossing over a vertex of the vehicle structure.

The design of the inlet is primarily concerned with supplying the combustor with favorable conditions. Variations in these conditions are important because the combustor is sensitive to temperature and pressure changes, meaning that the combustion efficiency can be reduced by lower pressure and temperature or increased by higher pressure and temperature.

For scram conditions, results show that burning early in the combustor, in the highest pressure and temperature regions, is best for thrust. It is possible to select more favorable conditions by considering the placement of the fuel injector with this in mind.

We suggest that performance of hypersonic vehicles be considered in a whole-flight-regime integrated sense, rather than a design point problem, since deviations from the design point are to be expected and since predictions of performance in regimes where boundary layer, wall heating and other properties are important is extremely difficult. While this may sacrifice some performance, it can make the vehicle more controllable by reducing discontinuities in thrust and give better results away from the design point.

\section{References}

\footnotetext{
${ }^{1}$ Bolender, M. A. and Doman, D. B., "Nonlinear Longitudinal Dynamical Model of an Air-Breathing Hypersonic Vehicle," Journal of Spacecraft and Rockets, Vol. 44, No. 2, 2007, pp. 374-387.

${ }^{2}$ Torrez, S. M., D. J. F. D. D. J. and Micka, D. J., "Scramjet Engine Model MASIV: Role of Mixing, Chemistry and Wave Interactions," AIAA-2009-4939.

${ }^{3}$ Torrez, S. M., D. J. F. D. D. J. B. M. A. and Doman, D. B., "Hypersonic Vehicle Thrust Sensitivity to Angle of Attack and Mach Number," AIAA-2009-6152.

${ }^{4}$ Bogar, T. J., Alberico, J. F., Johnson, D. B., Espinosa, A. M., and Lockwood, M. K., "Dual-Fuel Lifting Body Configuration Development," International Space Planes and Hypersonic Systems and Tehcnologies Conference, AIAA Paper 96-4592, 1996.

${ }^{5}$ Smart, M. K., "Optimization of Two-Dimensional Scramjet Inlets," Journal of Aircraft, Vol. 36, No. 2, 1999, pp. 430-433.

${ }^{6}$ Brown, M., Mudford, N. R., Neely, A. J., and Ray, T., "Robust Optimization of Two-Dimensional Scramjet Inlets," 14 th AIAA/AHI Space Planes and Hypersonic Systems and Technologies Conference, AIAA Paper 2006-8140, 2006.

${ }^{7}$ Chavez, F. R. and Schmidt, D. K., "Analytical Aeropropulsive/Aeroelastic Hypersonic-Vehicle Model with Dynamic Analysis," Journal of Guidance, Control, and Dynamics, Vol. 17, No. 6, 1994, pp. 1308-1319.
} 
${ }^{8}$ Frendreis, S. G. V., Skujins, T., and Cesnik, C. E. S., "Six-Degree-of-Freedom Simulation of Hypersonic Vehicles," AIAA Atmospheric Flight Mechanics Conference, 2009, AIAA Paper 2009-5601.

${ }^{9}$ Lind, C. A. and Lewis, M. J., "The Effect of Shock/Shock Interactions on the Design of Hypersonic Inlets," AIAA/SAE/ASME/ASEE 26th Joint Propulsion Conference, 1990.

${ }^{10}$ Micka, D. J., Torrez, S. M., and Driscoll, J. F., "Measurements and Modeling of the Heat Release Distribution in a Dual-mode Scramjet Combustor with Wall Fuel Injection," Proceedings of the 6th U.S. National Combustion Meeting, 2009.

${ }^{11}$ Micka, D. J. and Driscoll, J. F., "Combustion characteristics of a dual-mode scramjet combustor with cavity flameholder," Proc. Comb. Inst., Vol. 32, 2009, pp. 2397-2404.

${ }^{12}$ Shapiro, A. H., Dynamics and Thermodynamics of Compressible Fluid Flow, Ronald Press, NY, 1953.

${ }^{13}$ Torrez, S. M.; Scholten, N. M. D. D. J. F. B. M. D. D. and Oppenheimer, M. W., "A Scramjet Engine Model Including Effects of Precombustion Shocks and Dissociation," AIAA 2008-4619.

${ }^{14}$ Torrez, S. M. Driscoll, J. F. B. M. A. O. M. W. and Doman, D., "Effects of Improved Propulsion Modelling on the Flight Dynamics of Hypersonic Vehicle," 2008.

${ }^{15} \mathrm{O}$ 'Brien, T. F.; Starkey, R. P. and Lewis, M. J., "Quasi-One-Dimensional High-Speed Engine Model with Finite-Rate Chemistry," Journal of Propulsion and Power, Vol. Vol. 17, No. 6, 2001, pp. pp. 1366-1374.

${ }^{16}$ Hasselbrink, E. F. and Mungal, M. G., "Transverse jets and jet flames. Part 2. Velocity and OH field imaging," Journal of Fluid Mechanics, Vol. 443, 2001, pp. 27-68.

${ }^{17}$ Peters, N., "Laminar Diffusion Flamelet Models in Non-Premixed Turbulent Combustion," Progress in Energy Combustion Science, Vol. 10, 1984, pp. 319-339. 